\title{
Stability of the duality gap in linear optimization
}

\author{
M.A. Goberna · A.B. Ridolfi · V.N. Vera de Serio
}

Received: date / Accepted: date

\begin{abstract}
In this paper we consider the duality gap function $g$ that measures the difference between the optimal values of the primal problem and of the dual problem in linear programming and in linear semi-infinite programming. We analyze its behavior when the data defining these problems may be perturbed, considering seven different scenarios. In particular we find some stability results by proving that, under mild conditions, either the duality gap of the perturbed problems is zero or $+\infty$ around the given data, or $g$ has an infinite jump at it. We also give conditions guaranteeing that those data providing a finite duality gap are limits of sequences of data providing zero duality gap for sufficiently small perturbations, which is a generic result.
\end{abstract}

Keywords Linear programming · Linear semi-infinite programming · Duality gap function $\cdot$ Stability $\cdot$ Primal-dual partition

Mathematics Subject Classifications (2010) 90C05, 90C34, 90C31, 90C46

\section{Introduction}

Linear optimization consists in the minimization of a linear objective function subject to linear constraints. Here the duality gap plays an important role both for theoretical and for

This research was partially supported by MINECO of Spain and FEDER of EU, Grant MTM2014-59179C2-01 and SECTyP-UNCuyo Res. 4540/13-R.

M.A. Goberna

Department of Mathematics, University of Alicante, 03080 Alicante, Spain.

E-mail:mgoberna@ua.es

A.B. Ridolfi

Universidad Nacional de Cuyo, Facultad de Ciencias Aplicadas a la Industria, Facultad de Ciencias

Económicas, Mendoza, Argentina.

E-mail: abridolfi@gmail.com

V.N. Vera de Serio

Universidad Nacional de Cuyo, Facultad de Ciencias Económicas, Mendoza, Argentina

E-mail: virginia.vera@fce.uncu.edu.ar 
computational purposes. It is defined as the difference of the optimal value of the primal problem and of the optimal value of its dual problem, and it depends on the data defining these optimization problems. We will analyze the behavior of this duality gap when these data are perturbed; in doing this we will consider seven different scenarios related to the parameters that present admissible perturbations and the ones that remain fixed. To be more precise we need to establish some definitions and notation.

The primal problem we consider in this paper is given by

$$
P: \quad \inf _{x \in \mathbb{R}^{n}}\left\{c^{\prime} x: a_{t}^{\prime} x \geq b_{t} \forall t \in T\right\},
$$

where $T$ is an arbitrary set, $a_{t} \in \mathbb{R}^{n}, b_{t} \in \mathbb{R}$, for all $t \in T$, and $c \in \mathbb{R}^{n} ; c^{\prime}$ denotes the transpose of $c$, while $a_{t}^{\prime}$ is the transpose of $a_{t}$. We say that $P$ is a linear semi-infinite programming (LSIP) problem for arbitrary, possibly infinite, index set $T ; P$ is said to be a linear programming (LP) problem when $T$ is finite.

By defining $a: T \rightarrow \mathbb{R}^{n}, a(t)=a_{t}$, and $b: T \rightarrow \mathbb{R}, b(t)=b_{t}$, we identify the problem $P$ with the triplet $(a, b, c) \in\left(\mathbb{R}^{n}\right)^{T} \times \mathbb{R}^{T} \times \mathbb{R}^{n}$.

We associate with $P$ its Haar's dual problem $D$ which is the ordinary dual problem in LP (See (3) for its definition in the next section). We denote by $v(P)$ the optimal value of $P$, with $v(P)=+\infty$ when $P$ is an inconsistent problem, i.e. the feasible set of $P$ is empty. Similarly, $v(D)$ stands for the optimal value of $D$, with $v(D)=-\infty$ when $D$ is inconsistent. So, $v(P), v(D) \in \overline{\mathbb{R}}:=\mathbb{R} \cup\{ \pm \infty\}$. The dual problem $D$ has been defined in such a way that the weak duality theorem $v(D) \leq v(P)$ always holds. The duality gap of the pair $(P, D)$ is the difference $v(P)-v(D)$, if $v(P), v(D) \in \mathbb{R}$, and $+\infty$, otherwise.

Recall that, in LSIP, the duality gap is related to the viability of solving $P$ by discretization of the index set. More precisely, $v(P)=v(D)$ if and only if $P$ is discretizable, i.e., there exists a sequence $\left\{T_{r}\right\}$ of finite subsets of $T$ such that, denoting by $v\left(P_{r}\right)$ the optimal value of the LP subproblem

$$
P_{r}: \quad \inf _{x \in \mathbb{R}^{n}}\left\{c^{\prime} x: a_{t}^{\prime} x \geq b_{t} \forall t \in T_{r}\right\},
$$

we have $v(P)=\lim _{r} v\left(P_{r}\right)$ (see [1] and [10, Table 8.1]). Hence, the importance of studying the behavior of this duality gap.

Obviously the duality gap depends on the data $(a, b, c)$, which sometimes are not known with certainty. In this paper we are interested in the behavior of this gap under small perturbations of all, or some, of the data by considering different scenarios relative to the admissible perturbations. To this aim, we embed the nominal problem $P$, corresponding to a given triplet $(a, b, c)$, into a suitable topological space of admissible perturbed triplets, the so-called space of parameters $\Theta$. Denoting by $v^{P}(a, b, c)$ the optimal value of $P$ and by $v^{D}(a, b, c)$ the optimal value of $D$, the duality gap of $(a, b, c)$ is

$$
g(a, b, c):= \begin{cases}v^{P}(a, b, c)-v^{D}(a, b, c), & \text { if } v^{P}(a, b, c), v^{D}(a, b, c) \in \mathbb{R}, \\ +\infty, & \text { otherwise. }\end{cases}
$$

Observe that $v^{P}$ and $v^{D}$ are positively homogeneous, so that the duality gap function $g: \Theta \rightarrow$ $\mathbb{R}_{+} \cup\{+\infty\}$ is positively homogeneous too.

Some works, as [4] and [10], define the duality gap as 0 when the primal and dual problems have both the same infinite value (e.g. when one of them is inconsistent and the other is unbounded). Our present definition as $+\infty$ when $v^{P}(a, b, c)$ and $v^{D}(a, b, c)$ are not real numbers follows from the weak duality property and the usual criterion of defining $( \pm \infty)-( \pm \infty)=+\infty$ in the context of difference of convex (DC in short) functions when considering extended real-valued convex functions (see [17] and references therein). 
Many authors have studied, from different perspectives, the effect of small changes in some elements of the triplet on the feasible set, on the optimal set, and on the optimal value. For instance, [21] considers only perturbations of $b,[3]$ analyzes independent perturbations of $b$ and $c$, as well as perturbations of the pair $(a, b)$, [8] considers simultaneous perturbations of the whole triplet $(a, b, c)$, [13] estimates the effect on the optimal value of perturbations of the rim data $(b, c)$ in LP. On the other hand, [16], [5], and [6] deal with the generic uniqueness of optimal solutions in LP, the stability of the feasible set, and the stability of the optimal set, respectively, under perturbations of the technological data $a$. In particular, [9] studies the variation of the optimal value under directional perturbations of $a$ in LP (other works dealing with arbitrary perturbations of $a$ are mentioned there). It is worth noticing that, as observed by many authors (e.g. [11], [6] and [9]), the perturbations of $a$ are the hardest to deal with for both the stability and the sensitivity analyses. Moreover, there is a broad literature on the relationship between the semicontinuity, or the subdifferentiability, of the optimal value under perturbations of $b$ for non necessarily linear problems (e.g. [15]). To the authors' knowledge, this is the first work where a stability problem (in this case the stability of the duality gap) is simultaneously studied for perturbations of all nonempty subsets of the triplet $(a, b, c)$, which gives rise to seven different scenarios.

The term "gap function" has been first used in optimization by Auslender [2], who considered the variational inequality problem consisting of finding $x \in X$ such that

$$
F(x)^{\prime}(y-x) \geq 0 \quad \forall y \in X,
$$

where $X$ is a given nonempty closed convex subset of $\mathbb{R}^{n}$ and $F: \mathbb{R}^{n} \rightarrow \mathbb{R}^{n}$ is a given continuously differentiable monotone mapping. In fact, introducing the so-called gap function $G(x):=\max _{y \in X} F(x)^{\prime}(x-y),(2)$ turns out to be equivalent to the optimization problem $\inf _{x \in X} G(x)$. Different gap functions enjoying desirable properties have been proposed for the variational inequality problem (2) and for other related problems (e.g., vector variational, quasi-variational and pre-variational inequality problems, vector equilibrium problems, general equilibrium problems, etc.) in order to transform them into optimization problems to be solved with numerical optimization methods (typically, descent methods). Other gap functions have been introduced in convex optimization. For instance, [14] introduces a gap function expressing the duality gap of a given convex optimization problem in order to get a convex optimization algorithm.

In the framework of continuous infinite linear programming, with decision space $X$ being a Banach space, [22] introduces the gap function $g(b, c)$ defined only for those pairs $(b, c)$ providing primal and dual feasible problems (assumption guaranteeing that $g(b, c) \in$ $\mathbb{R}$, by the weak duality theorem) and keeping the gradient vectors $a_{t}$ fixed. It is worth observing that the LSIP problem $P$ in (1) does not satisfy the assumptions of [22] except in the case of continuous LSIP (where $T$ is a compact Hausdorff topological space and $A x:=\left(a_{t}^{\prime} x\right)_{t \in T}$, for all $x \in \mathbb{R}^{n}$, defines a continuous linear map from $\mathbb{R}^{n}$ to the Banach space $\mathscr{C}(T)$ ).

This paper is organized as follows: Section 2 introduces the dual problem and discusses known results about the basic primal-dual partition in the LSIP and LP frameworks. Section 3 provides characterizations of those parameters which maintain the consistency (or the inconsistency) of the corresponding primal (and dual) problem through sufficiently small perturbations of the data. Section 4 characterizes the parameters that maintain the simultaneous consistency of both the primal and the dual problems under small perturbations of the data in seven different scenarios. These results are applied to get conditions for the stability of the duality gap function and to give conditions guaranteeing that those data providing a finite duality gap are limits of sequences of data providing zero duality gap for sufficiently 
small perturbations. Moreover, this section gives sufficient conditions for a given non simultaneously (primal and dual) consistent parameter to maintain this property under small perturbations. Finally, Section 5 is devoted to the particular case of LP problems.

\section{The dual problem and the basic primal-dual partition}

We begin this section by introducing some necessary notation. Given a topological real vector space $X$ and $Y \subset X$, int $Y$, $\operatorname{rint} Y, \operatorname{cl} Y$, and bd $Y$ denote the interior, the relative interior, the closure, and the boundary of $Y$, respectively. Moreover, conv $Y$ stands for the convex hull of $Y$, whereas cone $Y:=\mathbb{R}_{+} \operatorname{conv} Y$ means the convex conical hull of $Y \cup\left\{0_{X}\right\}$, where $0_{X}$ denotes the null of $X$. For $X=\mathbb{R}^{n}$, we represent by $\geqq$ the usual partial order, by $\mathbf{0}_{n}$ the vector of all $0^{\prime} \mathrm{s}$, and by $\|\cdot\|_{\infty}$ the Chebyshev norm. Finally, $\lim _{k}$ stands for $\lim _{k \rightarrow \infty}$.

Now, we will recall known results from the LSIP and LP frameworks; all the primal problems $P$ are as in (1), with $(a, b, c) \in\left(\mathbb{R}^{n}\right)^{T} \times \mathbb{R}^{T} \times \mathbb{R}^{n}$.

The Haar's dual problem of $P$ is

$$
D: \sup _{\lambda \in \mathbb{R}_{+}^{(T)}}\left\{\sum_{t \in T} \lambda_{t} b_{t}: \sum_{t \in T} \lambda_{t} a_{t}=c\right\},
$$

where $\mathbb{R}_{+}^{(T)}$ is the positive cone in the space $\mathbb{R}^{(T)}$ of generalized finite sequences formed by all functions $\lambda \in \mathbb{R}^{T}$ such that $\lambda_{t}=0$ for all $t \in T$ except maybe for a finite number of indices. Observe that $D$ is a LSIP problem whenever $T$ is infinite and it is a LP problem otherwise. If $T=\{1, \ldots, m\}$, one has $\mathbb{R}^{(T)}=\mathbb{R}^{T}=\mathbb{R}^{m}$ and $\mathbb{R}_{+}^{(T)}=\mathbb{R}_{+}^{m}$, and $P$ is the LP problem (in canonical format) given by

$$
P: \inf _{x \in \mathbb{R}^{n}}\left\{c^{\prime} x: A x \geq b\right\}
$$

with $A(m \times n)$, while $D$ is its ordinary dual problem (in standard format), i.e.,

$$
D: \sup _{\lambda \in \mathbb{R}_{+}^{m}}\left\{b^{\prime} \lambda: A^{\prime} \lambda=c\right\}
$$

Other dual problems can be associated with $P$ following general schemes. For instance, the Lagrangian dual problem, or the dual problem in Rockafellar's sense ([19]), whose feasible set is $\mathbb{R}^{(T)}$. Nevertheless most of them are equivalent to the Haar's dual problem, which is the one that we consider here.

The space of parameters $\Theta$ is determined by the admissible perturbations and it can be partitioned in different ways. The basic primal (dual) partition is formed by the cone $\Pi_{c}$ $\left(\Delta_{c}\right)$ of parameters providing a consistent primal (dual) problem and the cone $\Pi_{i}\left(\Delta_{i}\right)$ of parameters providing an inconsistent primal (dual) problem. Related to the optimal values $v^{P}$ and $v^{D}$, the basic primal partition $\left\{\Pi_{c}, \Pi_{i}\right\}$, is formed by the sets of parameters with $v^{P}<+\infty$ and $v^{P}=+\infty$, respectively, while the basic dual partition $\left\{\Delta_{c}, \Delta_{i}\right\}$, is determined by the parameters with $v^{D}>-\infty$ and $v^{D}=-\infty$, respectively.

We denote $\Theta_{\alpha \beta}:=\Pi_{\alpha} \cap \Delta_{\beta}$, for $\alpha, \beta \in\{c, i\}$. The cells in Table 1 below, which are the intersections of the corresponding entries, indicate all the different states in the basic primal-dual partition. 


\begin{tabular}{|c|c|c|}
\hline & $\Delta_{c}$ & $\Delta_{i}$ \\
\hline$\Pi_{c}$ & $\Theta_{c c}$ & $\Theta_{c i}$ \\
\hline$\Pi_{i}$ & $\Theta_{i c}$ & $\Theta_{i i}$ \\
\hline
\end{tabular}

Table 1

Here the domain of the extended function $g$ is $\Theta_{c c}=\Pi_{c} \cap \Delta_{c}$. Since the entries of Table 1 are cones, the sets of the basic primal-dual partition are cones too. In particular, the domain of $g$ and its complement, $\Theta \backslash \Theta_{c c}$, are cones.

We associate with each triplet $(a, b, c) \in\left(\mathbb{R}^{n}\right)^{T} \times \mathbb{R}^{T} \times \mathbb{R}^{n}$ the first moment cone

$$
\text { cone }(a):=\text { cone }\left\{a_{t}, t \in T\right\}
$$

and the second moment cone

$$
\text { cone }(a, b):=\text { cone }\left\{\left(a_{t}, b_{t}\right), t \in T\right\} .
$$

We will also use the following notation for short:

$$
\operatorname{conv}(a):=\operatorname{conv}\left\{a_{t}, t \in T\right\} \quad \text { and } \quad \operatorname{conv}(a, b):=\operatorname{conv}\left\{\left(a_{t}, b_{t}\right), t \in T\right\},
$$

with cone $(a)=\mathbb{R}_{+} \operatorname{conv}(a)$ and cone $(a, b)=\mathbb{R}_{+} \operatorname{conv}(a, b)$. In LP, cone $(a)$ and cone $(a, b)$ are polyhedral convex cones while conv $(a)$ and conv $(a, b)$ are polytopes.

For any other parameter $\left(a^{1}, b^{1}, c^{1}\right) \in\left(\mathbb{R}^{n}\right)^{T} \times \mathbb{R}^{T} \times \mathbb{R}^{n}$ we will also use the brief notation cone $\left(a^{1}\right):=$ cone $\left\{a_{t}^{1}, t \in T\right\}$ and so on.

It is known ([10, Chapter 4$])$ that the set $\Pi_{c}$ of parameters providing primal consistent problems is characterized as

$$
(a, b, c) \in \Pi_{c} \Leftrightarrow\left(\mathbf{0}_{n}, 1\right) \notin \operatorname{clcone}(a, b),
$$

while the obvious characterization of $\Delta_{c}$ is

$$
(a, b, c) \in \Delta_{c} \Leftrightarrow c \in \text { cone }(a) .
$$

From the previous equivalences,

$$
(a, b, c) \in \Theta_{c c} \Leftrightarrow\left(\mathbf{0}_{n}, 1\right) \notin \operatorname{clcone}(a, b) \text { and } c \in \text { cone }(a) .
$$

In LP, the simultaneous consistency of $P$ and $D$ implies a zero duality gap together with the solvability of both problems. Nonetheless, this is not the case in LSIP where it is possible to have a finite positive duality gap for consistent problems $P$ and $D$, see [10, Example 2.1]. The classical LSIP duality theorems ([20], [4]) give conditions guaranteeing a zero duality gap together with the solvability of one of the coupled problems when $P$ and $D$ are both consistent. The first duality theorem establishes that $c \in$ rint cone $(a)$ implies $g(a, b, c)=0$ with $P$ solvable while the second duality theorem asserts that the closedness of cone $(a, b)+\mathbb{R}_{+}\left\{\left(\mathbf{0}_{n},-1\right)\right\}$ also entails $g(a, b, c)=0$ but now together with the solvability of $D$.

As we have stated, the space of parameters $\Theta$ is determined by the admissible perturbations, i.e., the elements of the triplet $(a, b, c)$ which can be perturbed. To avoid a somewhat cumbersome notation when dealing with different scenarios, we will always use the notation $\Theta$ for the parameter space which will have to be identified from the context; when all data are perturbable then $\Theta=\left(\mathbb{R}^{n}\right)^{T} \times \mathbb{R}^{T} \times \mathbb{R}^{n}$, when only $a$ and $b$ are perturbable while $c$ remains fixed then $\Theta=\left(\mathbb{R}^{n}\right)^{T} \times \mathbb{R}^{T} \times\{c\}$ which is identified with $\left(\mathbb{R}^{n}\right)^{T} \times \mathbb{R}^{T}$, and so on. In this paper we consider the seven parameter spaces introduced in Table 2 below. Observe that all of them are real linear spaces. 


\begin{tabular}{|c|c|c|}
\hline Scenario & Perturbable data & Parameter space $\Theta$ \\
\hline 1 & $(a, b, c)$ & $\left(\mathbb{R}^{n}\right)^{T} \times \mathbb{R}^{T} \times \mathbb{R}^{n}$ \\
\hline 2 & $(a, b)$ & $\left(\mathbb{R}^{n}\right)^{T} \times \mathbb{R}^{T}$ \\
\hline 3 & $(a, c)$ & $\left(\mathbb{R}^{n}\right)^{T} \times \mathbb{R}^{n}$ \\
\hline 4 & $(b, c)$ & $\mathbb{R}^{T} \times \mathbb{R}^{n}$ \\
\hline 5 & $a$ & $\left(\mathbb{R}^{n}\right)^{T}$ \\
\hline 6 & $b$ & $\mathbb{R}^{T}$ \\
\hline 7 & $c$ & $\mathbb{R}^{n}$ \\
\hline
\end{tabular}

Table 2

We choose topologies on the spaces of parameters corresponding to a reasonable measure of the size of the admissible perturbations. The box Chebyshev (pseudo) norm of $\theta=(a, b, c) \in\left(\mathbb{R}^{n}\right)^{T} \times \mathbb{R}^{T} \times \mathbb{R}^{n}$ is

$$
\|(a, b, c)\|_{\infty}:=\max \left\{\|c\|_{\infty}, \sup _{t \in T}\left\|\left(a_{t}, b_{t}\right)\right\|_{\infty}\right\} .
$$

The box Chebyshev (pseudo) distance between $\theta_{1} \in\left(\mathbb{R}^{n}\right)^{T} \times \mathbb{R}^{T} \times \mathbb{R}^{n}$ and $\theta_{2} \in\left(\mathbb{R}^{n}\right)^{T} \times$ $\mathbb{R}^{T} \times \mathbb{R}^{n}$ is $d_{\infty}\left(\theta_{1}, \theta_{2}\right):=\left\|\theta_{1}-\theta_{2}\right\|_{\infty}$. Obviously, $d_{\infty}$ describes the topology of the uniform convergence. All the different spaces of parameters shall be seen as subspaces of $\left(\mathbb{R}^{n}\right)^{T} \times$ $\mathbb{R}^{T} \times \mathbb{R}^{n}$ equipped with the corresponding restriction of $d_{\infty}$. In LP, where $T$ is finite, all these parameter spaces are connected. However, it is not the case in LSIP when $T$ is infinite because then it is well known that $\Theta$ is not a connected space with the box topology. The following example shows $\Theta_{c c}$ with different behaviors in different scenarios, for the same decision space $\mathbb{R}^{n}$ and (finite) index set $T$.

Example 1 Let us consider the simplest case that $n=|T|=1$, with $(a, b, c)=(0,1,0)$. It is easy to see that in

- scenario $1, \Theta_{c c}=\left(\{0\} \times \mathbb{R}_{-} \times\{0\}\right) \cup\left(\mathbb{R}_{++} \times \mathbb{R} \times \mathbb{R}_{+}\right) \cup\left(\mathbb{R}_{--} \times \mathbb{R} \times \mathbb{R}_{-}\right)$is connected but not convex,

- scenario $2, \Theta_{c c}=\mathbb{R}^{2} \backslash\left(\{0\} \times \mathbb{R}_{++}\right)$is also connected but not convex,

- scenario $3, \Theta_{c c}=\mathbb{R}^{2} \backslash(\{0\} \times \mathbb{R})$ is not even connected,

- scenario $4, \Theta_{c c}=\mathbb{R}_{-} \times\{0\}$ is convex,

- scenario $5, \Theta_{c c}=\mathbb{R} \backslash\{0\}$ is not connected,

- scenario $6, \Theta_{c c}=\mathbb{R}_{-}$is convex,

- scenario 7, $\Theta_{c c}=\emptyset$.

Concerning the function $g$, it is proper in scenarios $1-6$, with $g_{\mid \Theta_{c c}}$ identically zero, and it is improper in scenario 7 .

To the best of our knowledge, the stability of the duality gap function $g$ has not been studied up to now in the LP and LSIP framework. Let us start by considering its algebraic behavior where $\Theta_{c c}$ is always convex, i.e. in scenarios 4,6 , and 7 .

Scenario 6: Here $(a, c)$ is fixed and we assume that $c \in$ cone $(a)$ (otherwise $\Theta_{c c}=\emptyset$ ). Let $\alpha \in[0,1]$ and $b^{1}, b^{2} \in \Theta_{c c}$. Then there exist $x^{1}, x^{2} \in \mathbb{R}^{n}$ such that $a_{t}^{\prime} x^{1} \geq b_{t}^{1}$ and $a_{t}^{\prime} x^{2} \geq b_{t}^{2}$ for all $t \in T$. Since $a_{t}^{\prime}\left((1-\alpha) x^{1}+\alpha x^{2}\right) \geq(1-\alpha) b_{t}^{1}+\alpha b_{t}^{2}$ for all $t \in T, \Theta_{c c}$ is convex. The convexity of $v^{P}$ on $\Theta_{c c}$ can be easily obtained from this last property, while the convexity of $v^{D}$ is obvious. So, $g$ is a DC function.

Scenario 7: Assume that $\left(\mathbf{0}_{n}, 1\right) \notin \operatorname{clcone}(a, b)$ (otherwise $\Theta_{c c}=\emptyset$ ). Then $\Theta_{c c}=$ cone $(a)$ is a (non-empty) convex cone. It is known ([10, Theorem 8.1]) that $v^{D}$ is a proper concave function while $v^{P}$ coincides with its closure, so that it is concave too. Thus, $g$ is DC too. 
Consequently, in scenario 4, where $\Theta_{c c}$ is convex too (combine the arguments of scenarios 6 and 7), $g(\cdot, c)$ and $g(b, \cdot)$ are DC functions for any $c \in \mathbb{R}^{n}$ and $b \in \mathbb{R}^{T}$, respectively.

\section{Interiors of the sets of the basic primal and dual partitions}

In order to address the stability of the duality gap we need to discuss first a characterization of the interiors of the sets of the basic primal (dual) partition. We do this mostly by gathering known results . The following simple property will be useful:

Lemma 1 ([18, Lemma 3.4]) Let $z \in \mathbb{R}^{n}$ and $\left\{a_{t}, t \in T\right\} \subset \mathbb{R}^{n}$. If $z \in$ int cone (a), then there exists $\varepsilon>0$ such that for all $z^{1} \in \mathbb{R}^{n}$ and $\left\{a_{t}^{1}, t \in T\right\} \subset \mathbb{R}^{n}$ satisfying $\left\|z-z^{1}\right\|_{\infty}<\varepsilon$ and $\sup _{t \in T}\left\|a_{t}-a_{t}^{1}\right\|_{\infty}<\varepsilon$, we have $z^{1} \in$ int cone $\left(a^{1}\right)$.

From now on we will consider a given data $(a, b, c) \in\left(\mathbb{R}^{n}\right)^{T} \times \mathbb{R}^{T} \times \mathbb{R}^{n}$, and $\theta \in \Theta$ will stand for the corresponding parameter in the different scenarios according to Table 2.

3.1 Interiors of the sets $\Pi_{c}$ and $\Pi_{i}$

Lemma 2 (Interior of $\Pi_{c}$ ) If $\theta \in \Pi_{c}$, then the following statements hold:

(i)

$$
\theta \in \operatorname{int} \Pi_{c} \Leftrightarrow \mathbf{0}_{n+1} \notin \operatorname{clconv}(a, b),
$$

in scenarios 1, 2, 4, and 6 .

(ii) In scenarios 3 and 5, if $\sup _{t \in T} b_{t} \leq 0$, then int $\Pi_{c}=\Pi_{c}=\Theta$, otherwise (9) holds true.

(iii) int $\Pi_{c}=\Pi_{c}$ in scenario 7.

Proof (i) follows from [10, Theorem 6.1] and by observing that the proof there only uses perturbations of $b$, so that this equivalence holds in scenarios $1,2,4$, and 6 .

(ii) If $\sup _{t \in T} b_{t} \leq 0$, then $\emptyset \neq \Pi_{c}=\Theta$ in scenarios 3 and 5 (where $b$ remains fixed), and hence int $\Pi_{c}=\Pi_{c}$. Assume now that $\sup _{t \in T} b_{t}>0$. Then an application of [5, Theorem 4.2 $($ iii $) \Leftrightarrow(i v)$ ] gives that $\theta \in \operatorname{int} \Pi_{c}$ in scenarios 3 and 5 is equivalent to $\theta \in \operatorname{int} \Pi_{c}$ in scenario 1 , hence (9) follows.

(iii) In scenario 7, $(a, b)$ remains fixed, so $\Pi_{c}=\Theta$ whenever $\Pi_{c} \neq \emptyset$.

Remark 1 It is obvious that in scenario 7, where $(a, b)$ remains fixed, we always have $\Pi_{c}=\emptyset$ or $\Pi_{c}=\Theta$.

On the other hand, the inconsistency of some parameter, in any scenario, is maintained in some neighborhood when $\left(\mathbf{0}_{n}, 1\right) \in$ int cone $(a, b)$. We will discuss this condition and other sufficient conditions for the interior of $\Pi_{i}$.

The next lemma provides an easy to check sufficient condition for $\theta$ to be an interior point of $\Pi_{i}$, in any scenario.

Lemma 3 If $\sup _{t \in T} b_{t}=+\infty$ and $\sup _{t \in T}\left\|a_{t}\right\|<+\infty$, then $\theta \in \operatorname{int} \Pi_{i}$ in scenarios 1 to 7.

Proof Clearly in this case $\left(\mathbf{0}_{n}, 1\right) \in \operatorname{clcone}\left(a^{1}, b^{1}\right)$ for all data at a finite distance of $\theta$ in any scenario. Thus, (6) yields $\theta \in \operatorname{int} \Pi_{i}$. 
Lemma 4 (Interior of $\Pi_{i}$ ) If $\theta \in \Pi_{i}$, then the following statements hold:

(i) $\left(\mathbf{0}_{n}, 1\right) \in$ int cone $(a, b)$ is a sufficient condition for $\theta \in$ int $\Pi_{i}$ in any scenario.

(ii) If $\sup _{t \in T} b_{t}<+\infty$, then

$$
\theta \in \operatorname{int} \Pi_{i} \Leftrightarrow\left(\mathbf{0}_{n}, 1\right) \in \operatorname{int} \text { cone }(a, b),
$$

in scenarios $1,2,3$, and 5 .

(iii) If $\inf _{t \in T} b_{t}>0$, then $\theta \in \operatorname{int} \Pi_{i}$, in scenarios 4 and 6 .

(iv) int $\Pi_{i}=\Pi_{i}$ in scenario 7 .

Proof (i) In any scenario, the inconsistency of some parameter is maintained in some neighborhood when $\left(\mathbf{0}_{n}, 1\right) \in$ int cone $(a, b)$, on behalf of (6) and Lemma 1.

(ii) The proofs in [10, Theorem 6.3 and Lemma 6.5] only involve perturbations of $a$, so that

(10) is valid in scenarios $1,2,3$ and 5 provided that $\sup _{t \in T} b_{t}<+\infty$.

(iii) Let $0<\varepsilon<\inf _{t \in T} b_{t}$. As $\left(\mathbf{0}_{n}, 1\right) \in \operatorname{clcone}(a, b)$, we can write

$$
\left(\mathbf{0}_{n}, 1\right)=\lim _{k} \sum_{t \in T} \lambda_{t}^{k}\left(a_{t}, b_{t}\right)
$$

for some sequence $\left\{\lambda^{k}\right\} \subset \mathbb{R}_{+}^{(T)}$. In particular, we have

$$
\varepsilon \limsup _{k} \sum_{t \in T} \lambda_{t}^{k} \leq \lim _{k} \sum_{t \in T} \lambda_{t}^{k} b_{t}=1
$$

Thus $\liminf _{k} \sum_{t \in T} \lambda_{t}^{k}\left(b_{t}-\frac{\varepsilon}{4}\right)>\frac{1}{2}$ and so $\left(\mathbf{0}_{n}, 1\right) \in \operatorname{clcone}\left(a, b-\frac{\varepsilon}{4}\right)$. Now, for any $b^{1} \in \mathbb{R}^{(T)}$ with $\left\|b^{1}-b\right\|_{\infty}<\frac{\varepsilon}{4}$, it also holds that

$$
\left(\mathbf{0}_{n}, 1\right) \in \operatorname{clcone}\left(a, b^{1}\right)
$$

We conclude that $\theta \in \operatorname{int} \Pi_{i}$ in scenarios 4 and 6 , where $a$ is fixed.

(iv) In scenario 7, $(a, b)$ remains fixed, so $\Pi_{i}=\Theta$ whenever $\Pi_{i} \neq \emptyset$.

Lemma 5 (Interior of $\Pi_{i}$ in LP) Assume that $T$ is finite. Let $\theta \in \Pi_{i}$, then (10) characterizes $\theta \in \operatorname{int} \Pi_{i}$ in scenarios $1,2,3$, and 5 . Furthermore, $\Pi_{i}$ is open in scenarios 4,6 , and 7 .

Proof The cases of scenarios 1, 2, 3, and 5 follow from Lemma 4 (ii) because $\sup _{t \in T} b_{t}<$ $+\infty$ is always true. Now, assume we are in scenarios 4 or 6 and take some $\theta \in \Pi_{i}$. Then (6) implies that $\left(\mathbf{0}_{n}, 1\right) \in$ cone $(a, b)$ since this cone is closed because $T$ is a finite set. If $T=\{1, \ldots, m\}$, there exist nonnegative scalars $\lambda_{1}, \ldots, \lambda_{m}$ such that

$$
\sum_{t=1}^{m} \lambda_{t}\left(a_{t}, b_{t}\right)=\left(\mathbf{0}_{n}, 1\right)
$$

Let $\sum_{t=1}^{m} \lambda_{t}=\delta>0$. Then, for any $b^{1} \in \mathbb{R}^{m}=\mathbb{R}^{T}$ such that $\left\|b^{1}-b\right\|_{\infty}<\frac{1}{\delta}$ one has $\sum_{t=1}^{m} \lambda_{t} b_{t}^{1}>0$, so that $\left(\mathbf{0}_{n}, 1\right) \in \operatorname{cone}\left(a, b^{1}\right)$ and hence the associated parameter $\theta^{1} \in \Pi_{i}$. Thus $\theta$ is an interior point of $\Pi_{i}$. Finally, in scenario 7 we have only two possibilities $\Pi_{i}=\emptyset$ or $\Pi_{i}=\Theta$, thus $\Pi_{i}$ is always open in this case. 
3.2 Interiors of $\Delta_{c}$ and $\Delta_{i}$

Lemma 6 (Interior of $\Delta_{c}$ ) If $\theta \in \Delta_{c}$, then

(i)

$$
\theta \in \operatorname{int} \Delta_{c} \Leftrightarrow c \in \operatorname{int} \text { cone }(a)
$$

in scenarios 1, 3, 4, and 7 .

(ii) In scenarios 2 and 5 , if $c=\mathbf{0}_{n}$, then $\theta \in \operatorname{int} \Delta_{c}=\Delta_{c}=\Theta$, while for $c \neq \mathbf{0}_{n}$ (11) holds true.

(iii) int $\Delta_{c}=\Delta_{c}$ in scenario 6 .

Proof First observe that $c \in$ int cone $(a)$ is a sufficient condition for $\theta \in$ int $\Delta_{c}$ in any case as a straightforward consequence of Lemma 1 . So the implication $(\Leftarrow)$ in $(11)$ is always true in any scenario.

(i) $(\Rightarrow)$ has been shown in [12, Theorem 5] by using only perturbations of $c$.

(ii) $c$ remains fixed in scenarios 2 and 5. When $c=\mathbf{0}_{n}$, then it is clearly valid that $\Delta_{c}=\Theta$. Let $c \neq \mathbf{0}_{n}$. Assume that $\theta \in$ int $\Delta_{c}$ and that $c \notin$ int cone $(a)$. Then $c \in$ bdcone $(a)$ and so there exists an arbitrarily small vector $u \in \mathbb{R}^{n} \backslash\left\{\mathbf{0}_{n}\right\}$ such that $u^{\prime} c \leq 0$ and $u^{\prime} z \geq 0$ for all $z \in$ cone $(a)$. We will show that $c \notin$ cone $(a+u)$. Suppose by contradiction that $c \in$ cone $(a+u)$, then we can write $c=\mu(z+u)$, with $\mu>0$ as $c \neq \mathbf{0}_{n}$, and $z \in \operatorname{conv}(a)$. Multiplying by $u$ we get the following inconsistency:

$$
0 \geq u^{\prime} c=\mu u^{\prime}(z+u)=\mu\left(u^{\prime} z+\|u\|^{2}\right)>0 .
$$

So we have $c \notin$ cone $(a+u)$. Since $(a+u, b, c)$ defines a parameter $\theta^{u} \notin \Delta_{c}$ arbitrarily close to $\theta$, we get the contradiction $\theta \notin$ int $\Delta_{c}$.

(iii) In scenario $6(a, c)$ is fixed, thus if $\Delta_{c} \neq \emptyset$, then $\Delta_{c}=\Theta$.

Lemma 7 (Interior of $\Delta_{i}$ ) If $\theta \in \Delta_{i}$, then

(i)

$$
\boldsymbol{\theta} \in \operatorname{int} \Delta_{i} \Leftrightarrow \mathbf{0}_{n} \notin \operatorname{clconv}(a) \text { and } c \notin \operatorname{clcone}(a),
$$

in scenarios 1 and 3. Moreover, it is also valid in scenarios 2 and 5 when $\left\{a_{t}\right\}_{t \in T}$ is bounded. (ii)

$$
\theta \in \operatorname{int} \Delta_{i} \Leftrightarrow c \notin \operatorname{clcone}(a)
$$

in scenarios 4 and 7.

(iii) int $\Delta_{i}=\Delta_{i}$ in scenario 6 .

Proof (i) The equivalence (12) has already been shown in [18, Theorem 3.5 (ii)] by using perturbations of $a$ and $c$, which is valid only in scenarios 1 and 3. Nonetheless, that proof can be slightly modified in order to show the implication $(\Leftarrow)$ using only perturbations of $a$, so it also holds in scenarios 2 and 5.

Now, we only need to show the implication $(\Rightarrow)$ in scenarios 2 or 5 . Here $c$ is fixed in both scenarios. Assume that $\left\{a_{t}\right\}_{t \in T}$ is bounded. First, suppose that $\theta \in \operatorname{int} \Delta_{i}$ and $\mathbf{0}_{n} \in$ clconv $(a)$. For any $\varepsilon>0$, let $z=\sum_{t \in T} \lambda_{t} a_{t}$ with $\lambda \in \mathbb{R}_{+}^{(T)}$ and $\sum_{t \in T} \lambda_{t}=1$ be such that $\|z\|_{\infty}<\varepsilon$. Since $c \neq \mathbf{0}_{n}$, we can put

$$
a_{t}^{1}:=a_{t}+\frac{\varepsilon}{\|c\|_{\infty}} c-z
$$


Then $\left\|a_{t}^{1}-a_{t}\right\|_{\infty}<2 \varepsilon$ for all $t \in T$ and

$$
\sum_{t \in T} \lambda_{t} a_{t}^{1}=\frac{\varepsilon}{\|c\|_{\infty}} c
$$

which implies that $c \in \operatorname{cone}\left(a^{1}\right)$, a contradiction with $\theta \in \operatorname{int} \Delta_{i}$, thus $\mathbf{0}_{n} \notin \operatorname{clconv}(a)$. Now, suppose that $\theta \in \operatorname{int} \Delta_{i}$ and assume that $c \in \operatorname{clcone}(a)$. Let $M>0$ be such that conv $(a)$ is contained in the ball $\mathrm{B}\left(\mathbf{0}_{n} ; M\right)$ of radius $M$. As before, take any $\varepsilon>0$, and let $z=\sum_{t \in T} \lambda_{t} a_{t}$ with $\lambda \in \mathbb{R}_{+}^{(T)}$ be such that

$$
\|z\|_{\infty}>\frac{\|c\|_{\infty}}{2} \text { and }\|c-z\|_{\infty}<\frac{\varepsilon\|c\|_{\infty}}{2 M}
$$

Put

$$
\mu:=\sum_{t \in T} \lambda_{t}>0 \quad \text { and } \quad a_{t}^{1}:=a_{t}+\frac{c-z}{\mu} .
$$

Since $\left\|\frac{z}{\mu}\right\|_{\infty}<M$, we obtain

$$
\left\|a_{t}^{1}-a_{t}\right\|_{\infty}<\frac{1}{\mu} \frac{\varepsilon\|c\|_{\infty}}{2 M}<\frac{M}{\|z\|_{\infty}} \frac{\varepsilon\|c\|_{\infty}}{2 M}<\frac{2}{\|c\|_{\infty}} \frac{\varepsilon\|c\|_{\infty}}{2}=\varepsilon
$$

for all $t \in T$, and

$$
\sum_{t \in T} \lambda_{t} a_{t}^{1}=c
$$

which gives that $c \in$ cone $\left(a^{1}\right)$, a contradiction with $\theta \in \operatorname{int} \Delta_{i}$. Thus $c \notin$ clcone $(a)$.

(ii) In scenarios 4 and $7 a$ remains fixed. Since $\theta \in \Delta_{i}$, it follows that $c \notin$ cone $(a)$. If $c \notin$ cl cone $(a)$, clearly we can find a neighborhood $V$ of $c$ such that $c^{1} \notin \operatorname{clcone}(a)$ for all $c^{1} \in V$; hence $\theta \in \operatorname{int} \Delta_{i}$. On the other hand, if $c \in \operatorname{clcone}(a)$, then there is a sequence $\left\{c^{k}\right\} \subset$ cone $(a)$ that converges to $c$, which gives that $\theta \notin$ int $\Delta_{i}$.

(iii) In scenario $6(a, c)$ is fixed, thus $\Delta_{i}=\Theta$ whenever $\Delta_{i} \neq \emptyset$.

Remark 2 When the technological data $a$ is not bounded the implication $(\Rightarrow)$ in (i) in the previous lemma can be false in scenarios 2 and 5 as the following problem shows. Let $n=$ $2, T=\mathbf{N}$, and $P=\inf \left\{x_{2}: k x_{1}+k^{2} x_{2} \geq 0, k \in \mathbf{N}\right\}$, then $c=(0,1) \in$ clcone $\left\{\left(k, k^{2}\right), k \in \mathbf{N}\right\}$ and the associated parameter $\theta \in \operatorname{int} \Delta_{i}$. Nevertheless, the implication $(\Leftarrow)$ is always valid in these scenarios 2 and 5.

\section{Stability of the duality gap}

The partition $\left\{\operatorname{int} \Theta_{c c}\right.$, bd $\Theta_{c c}$, int $\left.\left(\Theta \backslash \Theta_{c c}\right)\right\}$ of $\Theta$ is related to our stability analysis of $g$. In fact, if $\theta \in$ bd $\Theta_{c c}$, then $g$ takes finite values and the value $+\infty$ on every neighborhood of $\theta$, i.e., $g$ has an infinite jump at $\theta$, which is an extreme form of instability. If $\theta \in \operatorname{int}\left(\Theta \backslash \Theta_{c c}\right)$, then $g$ is identically $+\infty$ in some neighborhood of $\theta$, in which case we say that $\infty$-stability holds. Finally, if $\theta \in \operatorname{int} \Theta_{c c}$, we know that $g$ has finite non-negative values in some neighborhood of $\theta$. In this section we show that under mild assumptions $g$ is identically 0 around $\theta$, situation described with the term 0-stability. Here we also complete the list of known characterizations of int $\Theta_{c c}$, and analyze the relationship between the continuity of $g$ and 0 -stability, which turns out to be a generic property on $\Theta_{c c}$. 
4.1 Stability of the duality gap under primal-dual consistency

The next result, characterizing int $\Theta_{c c}$, involves the following two conditions:

(C1) $\mathbf{0}_{n+1} \notin \operatorname{clconv}(a, b)$.

(C2) $c \in$ int cone $(a)$.

Observe that, by [10, Theorem 6.1], (C1) is equivalent to a primal Slater-type condition: there exists $\bar{x} \in \mathbb{R}^{n}$ (called strong Slater point) and $\varepsilon>0$ such that $a_{t}^{\prime} \bar{x} \geq b_{t}+\varepsilon$ for all $t \in T$. In turn, condition (C2) can also be seen as a dual Slater-type condition as it is guaranteed by the existence of $\lambda \in \mathbb{R}_{+}^{(T)}$ such that $c=\sum_{t \in T} \lambda_{t} a_{t}$ with $\left\{a_{t}: \lambda_{t}>0\right\}$ being a set of $n$ linearly independent vectors.

Theorem 1 (The interior of $\Theta_{c c}$ ) Table 3 characterizes the membership of an element of $\Theta_{c c}$ in its interior int $\Theta_{c c}$ for a given data $(a, b, c) \in\left(\mathbb{R}^{n}\right)^{T} \times \mathbb{R}^{T} \times \mathbb{R}^{n}$ :

\begin{tabular}{|c|c|c|}
\hline Scenario & Parameter & Characterization of its membership in int $\Theta_{c c}$ \\
\hline 1 & $(a, b, c)$ & $(C 1)$ and $(C 2)$ \\
\hline 2 & $(a, b)$ & $\begin{array}{l}\text { (C1), } \quad \text { if } c=\mathbf{0}_{n}, \\
(C 1) \text { and (C2), else. }\end{array}$ \\
\hline 3 & $(a, c)$ & $\begin{array}{l}(C 2), \quad \text { if } \sup _{t \in T} b_{t} \leq 0, \\
(C 1) \text { and }(C 2), \text { else. }\end{array}$ \\
\hline 4 & $(b, c)$ & $(C 1)$ and $(C 2)$ \\
\hline 5 & $a$ & $\begin{array}{ll}(C 1) \text { and }(C 2), & \text { if } c \neq \mathbf{0}_{n} \text { and } \sup _{t \in T} b_{t}>0, \\
(C 2), & \text { if } c \neq \mathbf{0}_{n} \text { and } \sup _{t \in T} b_{t} \leq 0, \\
(C 1), & \text { if } c=\mathbf{0}_{n} \text { and } \sup _{t \in T} b_{t}>0, \\
\text { It holds for sure, } & \text { if } c=\mathbf{0}_{n} \text { and } \sup _{t \in T} b_{t} \leq 0 .\end{array}$ \\
\hline 6 & $b$ & $(C 1)$ \\
\hline 7 & $c$ & $(C 2)$ \\
\hline
\end{tabular}

Proof The fact that int $\Theta_{c c}=\operatorname{int} \Pi_{c} \cap$ int $\Delta_{c}$ together with Lemmas 2 and 6 provide the proof.

Notice that, in Example 1, (C1) holds while (C2) fails. So, the parameter belongs to int $\Theta_{c c}$ exclusively in scenarios 2,5 , and 6.

Remark 3 Concerning scenario 5, it could be the case of having int $\Theta_{c c}=\Theta_{c c}$ even though $\sup _{t \in T} b_{t}>0$. As an example, let $b \in \mathbb{R}^{T}$ be a given function such that $\inf _{t \in T} b_{t}>0$ and $c=\mathbf{0}_{n}$. We then have that $(\mathrm{C} 1)$ holds for all $a \in\left(\mathbb{R}^{n}\right)^{T}$. In this case, given $a \in \Theta_{c c} \subset\left(\mathbb{R}^{n}\right)^{T}$, since $\sup _{t \in T} b_{t} \geq \inf _{t \in T} b_{t}>0$, Theorem 1 yields $a \in \operatorname{int} \Theta_{c c}$. This means that int $\Theta_{c c}=\Theta_{c c}$ not only when $c=\mathbf{0}_{n}$ and $\sup _{t \in T} b_{t} \leq 0$.

Theorem 2 (Characterization of 0 -stability) Let $\theta \in \Theta_{c c}$, with $\Theta$ being the parameter space for any scenario $k \in\{1,3,4,5,7\}$ or $k=2$ with $c \neq \mathbf{0}_{n}$. Then the following statements are equivalent to each other:

(i) $g$ is identically zero in some neighborhood of $\theta$.

(ii) $g$ is continuous at $\theta$.

(iii) $g$ is upper semi-continuous at $\theta$.

(iv) $\theta \in \operatorname{int} \Theta_{c c}$.

If $|T|<\infty$, then the four statements (i) - (iv) are equivalent for any $k \in\{1, \ldots, 7\}$. 
Proof $((i) \Rightarrow($ ii) $))$ and $[($ ii $) \Rightarrow($ iii $)]$ are trivial.

[(iii) $\Rightarrow\left(\right.$ iv) ] Given $\gamma>g(\theta)$, there exists a neighborhood $V_{\theta}$ of $\theta$ such that $\gamma>g\left(\theta_{1}\right)$ for any $\theta_{1} \in V_{\theta}$. Since $g$ is finite-valued on $V_{\theta}, V_{\theta} \subset \Theta_{c c}$ and the conclusion follows.

[(iv) $\Rightarrow(\mathrm{i})]$ According to Theorem 1, if $\theta \in \operatorname{int} \Theta_{c c}$, then $\theta$ satisfies (C2) in scenarios 1,2 (with $c \neq \mathbf{0}_{n}$ ), 3, 4, 5 (with $c \neq \mathbf{0}_{n}$ ), and 7. By Lemma 1, there exists a neighborhood $V_{\theta}$ of $\theta$ (w.l.o.g. we assume that $V_{\theta} \subset \Theta_{c c}$ ) such that (C2) also holds for any $\theta_{1} \in V_{\theta}$, in which case the first duality theorem yields $g\left(\theta_{1}\right)=0$ for all $\theta_{1} \in V_{\theta}$. The case of $c=\mathbf{0}_{n}$ in scenario 5 gives trivially $g=0$ on $\Theta_{c c}$.

Now we assume that $|T|<\infty$. Here [(iv) $\Rightarrow$ (i)] in any scenario with no extra condition because of the strong duality property.

The next example shows that $\theta \in \operatorname{int} \Theta_{c c}$ does not characterize the 0 -stability of $g$ in scenario 6 when $T$ is infinite.

Example 2 Let $T=\mathbb{N}$ and let $P$ be the problem

$$
\begin{array}{ll}
\inf _{x \in \mathbb{R}^{2}} & x_{2} \\
\text { s.t. } & -x_{1} \geq 0, \quad t=1, \\
& x_{2} \geq 0, \quad t=2, \\
& \quad-\frac{1}{t} x_{1}+x_{2} \geq 0, \quad t=3,4, \ldots . .
\end{array}
$$

Here $a: \mathbb{N} \rightarrow \mathbb{R}^{2}$ is given by $a_{1}=(-1,0)^{\prime}, a_{2}=(0,1)^{\prime}, a_{t}=\left(-\frac{1}{t}, 1\right)^{\prime}, t=3,4, \ldots ; b: \mathbb{N} \rightarrow$ $\mathbb{R}^{2}$ is the null function, and $c=(0,1)^{\prime}$. The unique solution of $\sum_{t \in \mathbb{N}} \lambda_{t} a_{t}=c$ in $\mathbb{R}_{+}^{(\mathbb{N})}$ is $\lambda=$ $(0,1,0, \ldots 0, \ldots)$. We will only allow for perturbations of $b$ (scenario 6), thus $\Theta=\mathbb{R}^{\mathbb{N}}$. Clearly

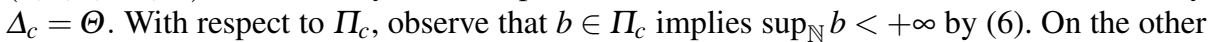
hand, $\mathbf{0}_{2} \notin \operatorname{conv}(a)$ provides $w \in \mathbb{R}^{2} \backslash\left\{\mathbf{0}_{2}\right\}$ and $\delta>0$ such that $a_{t}^{\prime} w \geq \delta$ for all $t \in \mathbb{N}$. If $b \in$ $\mathbb{R}^{\mathbb{N}}$ has an upper bound, then $\rho a_{t}^{\prime} w \geq \sup _{\mathbb{N}} b$ for all $t \in \mathbb{N}$ for $\rho$ sufficiently large, so that $b \in$ $\Pi_{c}$. Hence $\Pi_{c}=\left\{b \in \mathbb{R}^{\mathbb{N}}: \sup _{\mathbb{N}} b<+\infty\right\}$, and therefore $\Theta_{c c}=\left\{b \in \mathbb{R}^{\mathbb{N}}: \sup _{\mathbb{N}} b<+\infty\right\}$. We associate with each $\varepsilon \geq 0$ an element $b^{\varepsilon} \in \Theta=\mathbb{R}^{\mathbb{N}}$ such that

$$
b_{t}^{\varepsilon}= \begin{cases}-\varepsilon, & \text { if } t=2 \\ 0, & \text { otherwise }\end{cases}
$$

From Theorem 1 , we have $b^{\varepsilon} \in \operatorname{int} \Theta_{c c}$ for all $\varepsilon \geq 0$. Moreover, it is easy to see that the primal and dual feasible set of $b^{\varepsilon}$ are, respectively, $\mathbb{R}_{-} \times \mathbb{R}_{+}$and the singleton set $\{\lambda\}$, where $\lambda_{2}=1$ and $\lambda_{t}=0$ for all $t \in \mathbb{N} \backslash\{2\}$. Since $g\left(b^{\varepsilon}\right)=\varepsilon>0$ for all $\varepsilon>0, g$ is not $0-$ stable at $b^{\varepsilon}$ for all $\varepsilon \geq 0$.

Theorem 2 allows to assert that $g$ is 0 -stable at the elements of int $\Theta_{c c}$ in some scenarios, but not all of them. Now, observe that, in Example 1, int $\Theta_{c c}$ is dense in $\Theta_{c c}$ in all cases, except scenario 4 where int $\Theta_{c c}$ is empty. In fact, the next result shows that, under mild conditions, the 0 -stability property is generic in topological sense, i.e. it is satisfied at an open dense subset of the domain of $g$ (in fact, int $\Theta_{c c}$ is dense in $\Theta_{c c}$ ). See [7] for other concepts of genericity, e.g., in the sense of the measure theory, where the condition that the complement of the given open set w.r.t. the set of parameters enjoying the aimed property is required to have zero measure instead of having an empty interior. 
Lemma 8 Table 4 provides sufficient conditions guaranteeing the density of int $\Theta_{c c}$ in $\Theta_{c c}$ :

\begin{tabular}{|c|c|l|}
\hline Scenario & Parameter & Suff. cond. for int $\Theta_{c c}$ to be dense in $\Theta_{c c}$ \\
\hline 1 & $(a, b, c)$ & $|T| \geq n$ \\
\hline 2 & $(a, b)$ & $|T| \geq n$ or $c=\mathbf{0}_{n}$ \\
\hline 3 & $(a, c)$ & $|T| \geq n$ and $\sup _{t \in T} b_{t} \leq 0$ \\
\hline 4 & $(b, c)$ & dim cone $(a)=n$ \\
\hline 5 & $a$ & $|T| \geq n$ and $\sup _{t \in T} b_{t} \leq 0$ \\
\hline 6 & $b$ & No condition is needed \\
\hline 7 & $c$ & dim cone $(a)=n$ \\
\hline
\end{tabular}

Table 4

Moreover, $\Theta_{c c}$ is open in scenario 5 whenever $c=\mathbf{0}_{n}$ and $\left[\sup _{t \in T} b_{t} \leq 0\right.$ or $\left.\inf _{t \in T} b_{t}>0\right]$.

Proof We prove that the assumptions in Table 4 guarantee that $\Theta_{c c} \subset \operatorname{clint} \Theta_{c c}$ by analyzing the scenarios in descending order. Assume that $\Theta_{c c} \neq \emptyset$ and that a parameter in $\Theta_{c c}$ is given. Scenario 7: First, observe that $\Theta_{c c} \neq \emptyset$ implies that $\Theta_{c c}=$ cone $(a)$. The assumption entails int cone $(a) \neq \emptyset$, so that

$$
\Theta_{c c}=\text { cone }(a) \subset \operatorname{clcone}(a)=\operatorname{clint} \operatorname{cone}(a)=\operatorname{clint} \Theta_{c c} .
$$

Scenario 6: Let $b \in \Theta_{c c}$. Take any $\varepsilon>0$, we will show that $b-\varepsilon \in \operatorname{int} \Theta_{c c}$. Suppose, it is not the case, then from Theorem $1, \mathbf{0}_{n+1} \in \operatorname{clconv}(a, b-\varepsilon)=-\left(\mathbf{0}_{n}, \varepsilon\right)+\operatorname{clconv}(a, b)$, which implies that $\left(\mathbf{0}_{n}, 1\right) \in \operatorname{clcone}(a, b)$, i.e. $b \notin \Theta_{c c}$, a contradiction. Therefore $b-\varepsilon \in \operatorname{int} \Theta_{c c}$ for all $\varepsilon>0$, and so $b \in \operatorname{clint} \Theta_{c c}$.

Scenario 5: Suppose $|T| \geq n$ and $\sup _{t \in T} b_{t} \leq 0$. If $c=\mathbf{0}_{n}$ then $\Theta_{c c}=\Theta$ and the result is trivial. Assume $c \neq \mathbf{0}_{n}$. Since $c \in$ cone $(a)$, by Carathéodory's Theorem $c \in$ cone $\left\{a_{t}, t \in S\right\}$, for some $S \subset T,|S|=n$, and $a_{s_{1}} \neq a_{s_{2}}$ if $s_{1} \neq s_{2}, s_{1}, s_{2} \in S$. Then there exist arbitrarily small vectors $p_{t} \in \mathbb{R}^{n}, t \in S$, such that $c \in$ int cone $\left\{a_{t}+p_{t}, t \in S\right\}$, and $\left\{a_{t}+p_{t}, t \in S\right\}$ is a basis of $\mathbb{R}^{n}$. Defining $p_{t}=0$ for all $t \in T \backslash S$, one gets $c \in$ int cone $(a+p)$, with $p \in\left(\mathbb{R}^{n}\right)^{(T)} \subset\left(\mathbb{R}^{n}\right)^{T}$ such that $d_{\infty}(a, a+p)=\sup _{t \in T}\left\|p_{t}\right\|_{\infty}$ is arbitrarily small. Since $a+p \in \operatorname{int} \Theta_{c c}$ by Theorem $1, a \in$ clint $\Theta_{c c}$. The last affirmation about the openness of $\Theta_{c c}$ in scenario 5 follows immediately from Theorem 1 and Remark 3 .

Scenario 4: Let $(b, c) \in \Theta_{c c}$. On the one hand, by the argument of scenario $6, \mathbf{0}_{n+1} \notin$ clconv $\left(a, b-\frac{1}{r}\right)$ for all $r \in \mathbb{N}$. On the other hand, by the argument of scenario 7 , we can pick a sequence $\left\{c^{r}\right\} \subset$ int cone $(a)$ such that $c^{r} \rightarrow c$. Since $\left(b-\frac{1}{r}, c^{r}\right) \in \operatorname{int} \Theta_{c c}$ for all $r \in \mathbb{N}$ and $\left(b-\frac{1}{r}, c^{r}\right) \rightarrow(b, c)$, we have $(b, c) \in \operatorname{clint} \Theta_{c c}$.

Scenario 3: If $c \neq \mathbf{0}_{n}$, then the proof is the same as the one of scenario 5; in case $c=\mathbf{0}_{n}$ take the perturbation $p$ such that int cone $\left\{a_{t}+p_{t}, t \in S\right\} \neq \emptyset$ and choose $c^{p} \in$ int cone $\left\{a_{t}+p_{t}, t \in S\right\}$ such that $d_{\infty}\left((a, c),\left(a+p, c^{p}\right)\right)$ is arbitrarily small.

Scenario 2: First, as in scenario 6, observe that for any $\varepsilon>0$ we have $\mathbf{0}_{n+1} \notin \operatorname{clconv}(a, b-\varepsilon)$, which assures the existence of a neighborhood $V$ of $(a, b-\varepsilon)$ in int $\Pi_{c}$. If $c=\mathbf{0}_{n}$, reasoning as in the proof of scenario 6 we get that $(a, b-\varepsilon) \in \operatorname{int} \Theta_{c c}$ for all $\varepsilon>0$, so $(a, b) \in \operatorname{clint} \Theta_{c c}$. For $|T| \geq n$ and $c \neq \mathbf{0}_{n}$, we can copy the proof in scenario 5 to get an arbitrarily small perturbation $p$ of $a$ such that $(a+p, b-\varepsilon) \in \operatorname{int} \Theta_{c c}$, concluding that $(a, b) \in \operatorname{clint} \Theta_{c c}$.

Scenario 1: The proof can be found in [18, Theorem 4.1], where all the data are perturbed. 
Theorem 3 (Generic 0 -stability) Table 4 provides sufficient conditions guaranteeing the genericity of the 0 -stability of $g$ in $\Theta_{c c}$ for scenarios $1,2,3,4,5$ and 7. For $|T|<\infty$, it is also true in scenario 6.

Proof It follows immediately from Theorem 2 and Lemma 8.

Concerning Example 1, we have seen that int $\Theta_{c c}$ is dense in $\Theta_{c c}$ for all the scenarios (except scenario 4), even though Theorem 3 does not apply to scenarios 3 and 7. So, the assumptions in Theorem 3 are sufficient, but not necessary, for the genericity of the 0 -stability.

4.2 Stability of the duality gap under inconsistency

Now we analyze the $\infty$-stability, i.e. conditions for a given $\theta \in \Theta$ to be in the exterior of $\Theta_{c c}$ which is the set int $\left(\Theta \backslash \Theta_{c c}\right)$. First observe that int $\Pi_{i} \cup \operatorname{int} \Delta_{i} \subset \operatorname{int}\left(\Theta \backslash \Theta_{c c}\right)$ always. The example below shows that this inclusion can be stricted in scenarios $1,2,3$, and 5. On the other hand the next proposition proves the equality of these two sets in scenarios 4,6 , and 7.

Proposition 1 The equality $\operatorname{int}\left(\Theta \backslash \Theta_{c c}\right)=\operatorname{int} \Pi_{i} \cup \operatorname{int} \Delta_{i}$ holds true in scenarios 4,6 and 7.

Proof The proof is immediate for scenarios 6 and 7. So, we only consider scenario 4 . The inclusion $\supset$ is clearly true. Assume that $\theta=(b, c) \in \operatorname{int}\left(\Theta \backslash \Theta_{c c}\right)$ and $\theta \notin \operatorname{int} \Pi_{i} \cup \operatorname{int} \Delta_{i}$. Then, for any $k \in \mathbb{N}$ there exist $\theta_{k}^{\prime}=\left(b_{k}^{\prime}, c_{k}^{\prime}\right) \in \Pi_{c}$ and $\theta_{k}^{\prime \prime}=\left(b_{k}^{\prime \prime}, c_{k}^{\prime \prime}\right) \in \Delta_{c}$, with $\left\|\theta-\theta_{k}^{\prime}\right\|_{\infty}<1 / k$ and $\left\|\theta-\theta_{k}^{\prime \prime}\right\|_{\infty}<1 / k$. Thus, $\theta_{k}=\left(b_{k}^{\prime}, c_{k}^{\prime \prime}\right) \in \Theta_{c c}$, and $\left\|\theta-\theta_{k}\right\|_{\infty}<1 / k$, which contradicts the assumption $\theta=(b, c) \in \operatorname{int}\left(\Theta \backslash \Theta_{c c}\right)$.

The next example shows that the above proposition does not hold for the other scenarios.

Example 3 (int $\Pi_{i} \cup \operatorname{int} \Delta_{i} \subsetneq \operatorname{int}\left(\Theta \backslash \Theta_{c c}\right)$ ) Let $n=1$ and $T=\{0\} \cup \mathbb{N}$, and consider $a: T \rightarrow$ $\mathbb{R}$ such that $a_{0}=1, a_{k}=0$ for $k \in \mathbb{N}, b: T \rightarrow \mathbb{R}$ such that $b_{0}=0, b_{k}=\frac{1}{2}+\frac{1}{k}$ for $k \in \mathbb{N}$, and $c=-1$. Then the corresponding primal and dual problems are

$$
\begin{array}{cc}
P: \text { inf }-x & D: \sup \sum_{k=1}^{\infty} \lambda_{k}\left(\frac{1}{2}+\frac{1}{k}\right) \\
\text { s.t. } x \geq 0, \text { for } t=0, & \text { s.t. } \lambda_{0}+\sum_{k=1}^{\infty} \lambda_{k} .0=-1, \\
0 x \geq \frac{1}{2}+\frac{1}{k}, k \in \mathbb{N} . & \lambda_{0}, \lambda_{k} \geq 0, k \in \mathbb{N} .
\end{array}
$$

Consider any of the scenarios $1,2,3$, or 5 , and let $\theta=(a, b, c)$ (making a slight abuse of notation in scenarios 2,3 , and 5 where $b$ or $c$, or both, are fixed). Clearly $\theta \in \Theta \backslash \Theta_{c c}$. Since $0 \in \operatorname{clconv}(a)$, we have that $\theta \notin$ int $\Delta_{i}$. Moreover, $\sup b_{t}<+\infty$ and $(0,1) \notin$ int cone $(a, b)$ imply that $\theta \notin \operatorname{int} \Pi_{i}$. However, we will show that $\theta \in \operatorname{int}\left(\Theta \backslash \Theta_{c c}\right)$. In fact, let $\widehat{\theta}=(\widehat{a}, \widehat{b}, \widehat{c}) \in$ $\Theta$ with $\|\widehat{\theta}-\theta\|_{\infty}<\frac{1}{4}$. If for some $k \in T$ the corresponding $\widehat{a}_{k}$ is negative, then we have that $(0,1) \in \operatorname{clcone}(\widehat{a}, \widehat{b})$ and thus $\widehat{\theta} \in \Theta \backslash \Theta_{c c}$. Otherwise, if $\widehat{a}_{k} \geq 0$ for all $k \in T$, then $\widehat{c} \notin$ cone $(\widehat{a})$ and so $\widehat{\theta} \in \Theta \backslash \Theta_{c c}$. Hence $\theta \in \operatorname{int}\left(\Theta \backslash \Theta_{c c}\right)$. 
Therefore, we may have

$$
\operatorname{int} \Pi_{i} \cup \operatorname{int} \Delta_{i} \varsubsetneqq \operatorname{int}\left(\Theta \backslash \Theta_{c c}\right)
$$

in the scenarios $1,2,3$, and 5 .

Now we provide sufficient conditions for the membership of $\theta \in \Theta \backslash \Theta_{c c}$ in $\operatorname{int}\left(\Theta \backslash \Theta_{c c}\right)$. The next result involves the following conditions:

(S0) $\left\{\begin{array}{l}\left(\mathbf{0}_{n}, 1\right) \in \text { int cone }(a, b), \\ \text { or } \\ \sup _{t \in T}\left\|a_{t}\right\|_{\infty}<+\infty \text { and } \sup _{t \in T} b_{t}=+\infty,\end{array}\right.$

(S1) $\quad \mathbf{0}_{n} \notin \operatorname{clconv}(a)$ and $c \notin \operatorname{clcone}(a)$.

(S2) $\left\{\begin{array}{l}\left(\mathbf{0}_{n}, 1\right) \in \operatorname{clcone}(a, b) \text { and }\left[\inf _{t \in T} b_{t}>0 \text { or }|T|<\infty\right], \\ \text { or } \\ c \notin \operatorname{clcone}(a) .\end{array}\right.$

(S3) $\left\{\begin{array}{l}\left(\mathbf{0}_{n}, 1\right) \in \operatorname{clcone}(a, b) \text { and }\left[\inf _{t \in T} b_{t}>0 \text { or }|T|<\infty\right], \\ \text { or } \\ c \notin \text { cone }(a) .\end{array}\right.$

(S4) $\left\{\begin{array}{l}\left(\mathbf{0}_{n}, 1\right) \in \operatorname{clcone}(a, b), \\ \text { or } \\ c \notin \text { clcone }(a) .\end{array}\right.$

Notice that a parameter that satisfies any of the conditions (S0) to (S4) is in $\Theta \backslash \Theta_{c c}$ for the seven scenarios. The following theorem shows that the conditions stated in (S1) to (S4) imply $\theta \in \operatorname{int} \Pi_{i} \cup$ int $\Delta_{i} \subset \operatorname{int}\left(\Theta \backslash \Theta_{c c}\right)$ in the different scenarios 1-7.

Theorem 4 (The exterior of $\Theta_{c c}$ ) Let $(a, b, c) \in\left(\mathbb{R}^{n}\right)^{T} \times \mathbb{R}^{T} \times \mathbb{R}^{n}$ be given. The following statements hold:

(i) (SO) is a sufficient condition for the membership of an element of $\Theta$ in $\operatorname{int}\left(\Theta \backslash \Theta_{c c}\right)$ in any scenario.

(ii) (S1) is a sufficient condition for the membership of an element of $\Theta$ in $\operatorname{int}\left(\Theta \backslash \Theta_{c c}\right)$ in scenarios 1, 2, 3, and 5 .

(iii) Table 5 provides characterizations of $\operatorname{int}\left(\Theta \backslash \Theta_{c c}\right)$ in scenarios 4 , 6, and 7.

\begin{tabular}{|c|c|c|}
\hline Scenario & Parameter & $\begin{array}{l}\text { Characterization of its } \\
\text { membership to int }\left(\Theta \backslash \Theta_{c c}\right)\end{array}$ \\
\hline 4 & $(b, c)$ & $(S 2)$ \\
\hline 6 & $b$ & $(S 3)$ \\
\hline 7 & $c$ & $(S 4)$ \\
\hline \multicolumn{2}{|c|}{ Table 5 } \\
\hline
\end{tabular}

Proof It follows immediately from Proposition 1 and the characterizations of int $\Pi_{i}$ and of int $\Delta_{i}$ given in Lemmas 4, 5 and 7 (taking into account Remark 2), because int $\Pi_{i} \cup \operatorname{int} \Delta_{i} \subset$ $\operatorname{int}\left(\Theta \backslash \Theta_{c c}\right)$ in any scenario. 


\section{Stable duality gap in linear programming}

In this section we will consider the specific case of $|T|<\infty$, where $P$ and $D$ are both ordinary LP problems. We have seen that, when $|T|<\infty, g$ is the indicator function of $\Theta_{c c}$, so that it is $0-$ stable at $\theta$ if and only if $\theta \in \operatorname{int} \Theta_{c c}$. It is clear that all the results valid in LSIP are also true for LP, nonetheless there are specific properties that are valid when all the parameter spaces are finite-dimensional. For instance, we know that $\Theta_{c c}$ in scenarios 4,6 , and 7 is a convex subset of $\Theta$ (which is now some Euclidean space $\mathbb{R}^{s}$ ), so it is always true that $\Theta_{c c} \subset$ clrint $\Theta_{c c}$, where rint $\Theta_{c c}$ is the relative interior of $\Theta_{c c}$, complementing in this way the genericity result provided by Theorem 3. Moreover, the (Lebesgue) measure of the set $\Theta_{c c} \backslash$ int $\Theta_{c c}$ is always zero in these scenarios because of the convexity of $\Theta_{c c}$, so the 0 -stability property of $g$ is also generic in the sense of [7] in scenarios 4, 6 and 7 .

To be more precise, this section is about the reformulation of LP problems in order to apply the results of Section 3 allowing to classify a given $\theta \in \Theta_{c c}$ as an element of the partition $\left\{\right.$ int $\Theta_{c c}$, bd $\Theta_{c c}$, int $\left.\left(\Theta \backslash \Theta_{c c}\right)\right\}$, and the way to check the assumptions of such results, i.e. modeling and computational issues on the stability of the duality gap in LP.

\subsection{Modeling issues}

Any LP problem with uncertain data can be reformulated in canonical and standard formats (as $P$ in (4) and $D$ in (5)), with $X=\mathbb{R}^{n}, A(m \times n)$ a real matrix identified with $a \in \mathbb{R}^{n m}$, $b \in \mathbb{R}^{m}$, and $c \in \mathbb{R}^{n}$ by means of well-known algebraic transformations. In some cases (as in Example 4 below) the results in Section 4 can be directly applied to the given LP problem but, in general, these reformulations do not fit perfectly into one of the scenarios described in Table 2, so that the mentioned results only provide sufficient conditions for the classification of $\theta$ into one of the elements of the partition $\left\{\operatorname{int} \Theta_{c c}\right.$, bd $\Theta_{c c}$, int $\left.\left(\Theta \backslash \Theta_{c c}\right)\right\}$. Examples 5 and 6 below illustrate this reformulation task.

In this subsection we denote by $\Theta^{k}$ the space of parameters corresponding to scenario $k$. We also represent by $\mathbf{1}_{p}$ the $p \times 1$ vector of all $1^{\prime} \mathrm{s}$.

Example 4 In this linear assignment problem the variable $y_{i j} \in\{0,1\}$ represents the assignment of agent $i$ to task $j, i, j=1, \ldots, p$, taking value 1 (with cost $\gamma_{i j} \geq 0$ ) if the assignment is done and 0 otherwise. The problem can be formulated as

$$
\begin{aligned}
D_{L}: \inf & \sum_{i, j=1, \ldots, p} \gamma_{i j} y_{i j} \\
\text { s.t. } & \sum_{i=1, \ldots, p} y_{i j}=1, \forall j=1, \ldots, p, \\
& \sum_{j=1, \ldots, p} y_{i j}=1, \forall i=1, \ldots, p, \\
& y_{i j} \geq 0, i, j=1, \ldots, p,
\end{aligned}
$$

where we have replaced the Boolean condition $y_{i j} \in\{0,1\}$ by $y_{i j} \geq 0$ because the constraint matrix $A\left(p^{2} \times 2 p\right)$ is totally unimodular. Obviously, $D_{L}$ is a particular instance of the problem $D$ in (5), with $m=p^{2}, n=2 p, c=\mathbf{1}_{2 p}$, and $b_{i j}=-\gamma_{i j}, i, j=1, \ldots, p$. Observe that the unique perturbable data in $D_{L}$ are the costs $\gamma_{i j}$, i.e., we are in scenario 6, with $\Theta=\Theta^{6}=\mathbb{R}^{p^{2}}$. If we order the $p^{2}$ indices $t=(i, j)$ with the lexicographical order as: 
$(1,1),(1,2), \ldots,(1, p),(2,1), \ldots(p, p)$, then the transpose $A^{\prime}$ of $A$ can be written in the form

$$
A^{\prime}=\left[\begin{array}{ccccc}
I_{p} & I_{p} & \ldots & \ldots & I_{p} \\
\mathbf{1}_{p}^{\prime} & \mathbf{0} & \cdots & \cdots & \mathbf{0} \\
\mathbf{0} & \mathbf{1}_{p}^{\prime} & \cdots & \cdots & \mathbf{0} \\
\vdots & \vdots & \vdots & \vdots & \vdots \\
\mathbf{0} & \mathbf{0} & \cdots & \cdots & \mathbf{1}_{p}^{\prime}
\end{array}\right]
$$

where $I_{p}$ is the unit $p \times p$ matrix. Observe that the $(i, i)$-column of $A^{\prime}$ is the $2 p$-vector $\left(e_{i}, e_{i}\right)^{\prime}$, where $e_{i}$ is the canonical $p$-vector. Then $b \in \Delta_{c}$ because $c$ is the sum of all the $(i, i)-$ columns of $A^{\prime}$. The fact that $\sup b_{i j} \leq 0$, gives that $P_{L}$ is consistent, and hence $b \in \Theta_{c c}$. Thus, Theorem 1 applies directly to obtain that

$$
g \text { is } 0 \text {-stable at } \theta \Leftrightarrow(\mathrm{C} 1) .
$$

Now, it is clear that no convex combination of the $a_{i j}$ 's is null, so $(\mathrm{C} 1)$ is valid and we obtain the 0 -stability of $g$ at $\theta$. (We could also have obtained this result from an application of the theory of totally unimodular matrices which assures us that $D_{L}$ is solvable).

Example 5 Allocating $q$ scarce resources among $n$ production lines can be formulated as

$$
D_{R}: \sup _{y \in \mathbb{R}_{+}^{n}}\left\{\sum_{j=1, \ldots, n} p_{j} y_{j}: W^{\prime} y \leq c\right\}
$$

where $y_{j}$ represents the production level of the $j$-th good (a decision variable), $p_{j}$ the price of the $j$-th good, $c_{i} \in \mathbb{R}_{+}$the available amount of the $i$-th resource, and $W(n \times q)$ the technological matrix (with non-negative entries). Introducing a slack variable $z \in \mathbb{R}_{+}^{q}$, and defining $\lambda^{\prime}:=\left(y^{\prime}, z^{\prime}\right), b^{\prime}:=\left(p_{1}, \ldots, p_{n}, 0, \ldots, 0\right) \in \mathbb{R}^{n+q}$, and $A^{\prime}:=\left[W^{\prime} \mid I_{q}\right]$, where $I_{q}$ denotes the unit $q \times q$ matrix, $D_{R}$ can be expressed as $D$ in (5), with $m=n+q$. The corresponding primal problem is consistent because of the assumption of non-negative entries in $W$. Since $D_{R}$ is also consistent, $\theta \in \Theta_{c c}$.

(i) If only the resource vector $c$ is uncertain, then $\Theta=\Theta^{7}=\mathbb{R}^{q}$ and, by Theorem 1,

$$
g \text { is } 0 \text {-stable at } \theta \Leftrightarrow(\mathrm{C} 2) \text {. }
$$

(ii) If, additionally, the price vector $b$ is uncertain too, then $\{b\} \times \Theta^{7} \varsubsetneqq \Theta \varsubsetneqq \Theta^{4}=\mathbb{R}^{n+2 q}$ (observe that only the first $n$ components of $b$ can be perturbed) and Theorem 1 yields

$$
g \text { is } 0 \text {-stable at } \theta \Leftrightarrow(\mathrm{C} 1) \text { and (C2). }
$$

Now, it is easy to observe that $(\mathrm{C} 1)$ is always true in this framework when $W$ has non-null rows or all the prices are positive, hence in this case we also obtain the equivalence (13) between the 0 -stability of $g$ at $\theta$ and (C2).

(iii) If all the data in $D_{R}, c, p$, and $W$, are perturbable, then $\{a\} \times\{b\} \times \Theta^{7} \varsubsetneqq \Theta \varsubsetneqq \Theta^{1}=$ $\mathbb{R}^{(n+q) q+n+2 q}$ and the conclusion is the same as in (ii).

Example 6 Example 1.1 in [6], dealing with two person, finite, zero-sum games, involves a particular LP problem $P_{G}$ of the form

$$
\begin{aligned}
& P_{G}: \text { inf } \mathbf{1}_{n}^{\prime} x \\
& \text { s.t. } U^{\prime} x \geqq \mathbf{1}_{m}, \quad x \geqq \mathbf{0}_{n},
\end{aligned}
$$


where $U(n \times m)$ denotes the pay-off matrix, whose entries can be assumed w.l.o.g. to be positive but subject to small perturbations while the remaining data are deterministic. Obviously, $P_{G}$ can be formulated as $P$ in (4), with $A^{\prime}=\left[U \mid I_{n}\right], b^{\prime}=\left(\mathbf{1}_{m}^{\prime}, \mathbf{0}_{n}^{\prime}\right)$ and $c=\mathbf{1}_{n}$. Since $P_{G}$ is solvable, $\theta \in \Theta_{c c}$. Moreover, the unique perturbable data are the entries of $U$, so that $\Theta \varsubsetneqq \Theta^{5}=\mathbb{R}^{(m+n) n}$ and Theorem 1 yields the sufficient condition:

$$
(\mathrm{C} 1) \text { and }(\mathrm{C} 2) \Rightarrow g \text { is } 0 \text {-stable at } \theta \text {. }
$$

It is clear that (C2) is satisfied because $c=\mathbf{1}_{n}$ and cone $(a)=\mathbb{R}_{+}^{(m+n) n}$. Furthermore, $(\mathrm{C} 1)$ is also valid by a simple inspection of the form of $A$ and $b$. Therefore $g$ is 0 -stable at $\theta$.

\subsection{Computational issues}

Denote by $M$ a $p \times n$ matrix without zero rows such that cone $(a)=\left\{x \in \mathbb{R}^{n}: M x \geqq \mathbf{0}_{p}\right\}$ and by $N$ a $q \times(n+1)$ matrix without zero rows such that cone $(a, b)=\left\{x \in \mathbb{R}^{n+1}: N x \geqq \mathbf{0}_{q}\right\}$ ( $p$ and $q$ are natural numbers). According to (8),

$$
(a, b, c) \in \Theta_{c c} \Leftrightarrow N\left(\begin{array}{c}
\mathbf{0}_{n} \\
1
\end{array}\right) \supsetneqq \mathbf{0}_{q} \text { and } M c \geqq \mathbf{0}_{p} .
$$

(Example 7 below illustrates the effective computation of $M$ and $N$.) Recall that (C1) is the Slater condition in LP.

Theorems 1 and 4 are still valid if we replace the geometric conditions (C1), (C2), (S0), ..., (S4) by the following vector inequalities and/or conditions expressed in terms of optimal values of LP problems:

(C1) $\sup \left\{x_{n+1}: A x-x_{n+1} \mathbf{1}_{m} \geqq b\right\}>0$.

(C2) $M c>\mathbf{0}_{p}$.

(S0) $N\left(\begin{array}{c}\mathbf{0}_{n} \\ 1\end{array}\right)>\mathbf{0}_{q}$.

(S1) $\sup \left\{x_{n+1}: A x-x_{n+1} \mathbf{1}_{m} \geqq \mathbf{0}_{m}\right\}=+\infty$ and $M c \nsupseteq \mathbf{0}_{p}$.

$$
(\mathrm{S} 2) \equiv(\mathrm{S} 3) \equiv(\mathrm{S} 4)\left\{\begin{array}{l}
N\left(\begin{array}{c}
\mathbf{0}_{n} \\
1
\end{array}\right) \geqq \mathbf{0}_{q}, \\
\text { or } \\
M c \nsupseteq \mathbf{0}_{p} .
\end{array}\right.
$$

All matrices and vectors in the above conditions, except $M$ and $N$, are expressed in terms of the data defining $P$ and $D$. Concerning the matrices $M$ and $N$, they can also be obtained from the data by eliminating $\lambda_{1}, \ldots, \lambda_{m}$ from the linear systems

$$
\left\{x=\sum_{t=1, . ., m} \lambda_{t} a_{t} ; \lambda_{t} \geq 0, t=1, . ., m\right\}
$$

and

$$
\left\{\left(x, x_{n+1}\right)=\sum_{t=1, . ., m} \lambda_{t}\left(a_{t}, b_{t}\right) ; \lambda_{t} \geq 0, t=1, . ., m\right\},
$$

respectively. Thus, all the above conditions are checkable. 
Example 7 Let

$$
A=\left[\begin{array}{rrr}
-1 & 0 & -2 \\
0 & 1 & 1 \\
0 & 1 & 0 \\
1 & 0 & 0 \\
0 & 1 & -1
\end{array}\right] \quad \text { and } b=\left(\begin{array}{r}
1 \\
0 \\
-1 \\
0 \\
0
\end{array}\right)
$$

We start by computing the auxiliary matrices $M$ and $N$. Gauss elimination of $\lambda_{1}, \lambda_{3}$, and $\lambda_{4}$ in (5.2) yields the inequality system

$$
\left\{2 x_{1}-x_{3}+\lambda_{2}-\lambda_{5} \geq 0 ; x_{2}-\lambda_{2}-\lambda_{5} \geq 0 ;-x_{3}+\lambda_{2}-\lambda_{5} \geq 0 ; \lambda_{2} \geq 0 ; \lambda_{5} \geq 0\right\}
$$

and Fourier elimination of $\lambda_{2}, \lambda_{5}$ from (15) provides the aimed linear representation of the first moment cone

$$
\text { cone }(a)=\left\{x \in \mathbb{R}^{3}: 2 x_{1}+x_{2}-x_{3} \geq 0 ; x_{2}-x_{3} \geq 0 ; x_{2} \geq 0\right\}
$$

so that

$$
M=\left[\begin{array}{rrr}
2 & 1 & -1 \\
0 & 1 & -1 \\
0 & 1 & 0
\end{array}\right]
$$

In a similar way one gets

$$
N=\left[\begin{array}{rrrr}
3 & 1 & -1 & 1 \\
0 & 1 & -1 & 1 \\
0 & 1 & -1 & -2 \\
0 & 2 & 1 & 2
\end{array}\right],
$$

with $N\left(\begin{array}{c}\mathbf{0}_{3} \\ 1\end{array}\right)=(-1,-1,2,-2)^{\prime} \nsupseteq \mathbf{0}$, which gives the consistency of the primal problem for any $c$. Now we consider different $c$ 's in the triplet $(a, b, c)$ :

(i) $c=(0,3,-2)^{\prime}$. As $M c=(5,5,3) \geqq \mathbf{0}_{3}$, we have $\theta \in \Theta_{c c}$. Moreover, since $\sup \left\{x_{4}: A x-x_{4} \mathbf{1}_{5} \geqq b\right\}=+\infty,(\mathrm{C} 1)$ and (C2) hold, so that $\theta \in$ int $\Theta_{c c}$ and hence $g$ is 0 stable at $\theta$ in all scenarios.

(ii) $c=(0,1,1)^{\prime}$. We still have $\theta \in \Theta_{c c}$, but now $M c=(0,0,1)^{\prime} \geqq \mathbf{0}_{3}$ has null components. Thus, since (C1) holds while (C2) fails, Theorem 1 allows to conclude that $\theta \in \operatorname{int} \Theta_{c c}$ only in scenario 6 (under perturbations of $b$ ). Then $g$ is 0 -stable at $\theta$ in scenario 6 and it is not 0 -stable at $\theta$ in the other scenarios.

(iii) $c=(-1,0,0)^{\prime}$. Now $M c=(-2,0,0)^{\prime} \nsupseteq \mathbf{0}_{3}$, so that $\theta \in \Theta \backslash \Theta_{c c}$. Since

$$
\sup \left\{x_{4}: A x-x_{4} \mathbf{1}_{5} \geqq \mathbf{0}_{5}\right\}=+\infty,
$$

(S1)-(S4) hold. Hence, by Theorem 4, $\theta \in \operatorname{int}\left(\Theta \backslash \Theta_{c c}\right)$ for any scenario. 


\section{References}

1. Astafev, N.N.: On Infinite Systems of Linear Inequalities in Mathematical Programming (in Russian), Nauka, Moscow (1991).

2. Auslender, A.: Optimisation: Méthodes Numériques, Masson, Paris (1976).

3. Brosowski, B.: Parametric Semi-Infinite Optimization, Verlag Peter Lang, Frankfurt am Main - Bern (1982).

4. Charnes, A., Cooper, W.W., Kortanek, K.O.: On representations of semi-infinite programs which have no duality gaps, Management Sci., 12 pp. 113-121 (1965).

5. Daniilidis, A., Goberna, M.A., Lopez, M.A., Lucchetti, R.: Lower semicontinuity of the solution set mapping of linear systems relative to their domains, Set-Valued Var. Anal., 21, pp. 67-92 (2013).

6. Daniilidis, A., Goberna, M.A., Lopez, M.A., Lucchetti, R.: Stability in linear optimization under perturbations of the left-hand side coefficients, Set-Valued Var. Anal., 23, pp. 737-758 (2015).

7. Duer, M., Jargalsaikhan, B., Still,G.: Genericity results in linear conic programming -a tour d'horizon, prepint. http://wwwhome.math.utwente.nl/_stillgj/sip/rev_bds.pdf (2015).

8. Fischer, T.: Contributions to semi-infinite linear optimization, Meth. Verf. Math. Phys., 27, pp. 175-199 (1983).

9. Ghaffari-Hadigheh, A., Mehanfar, N.: Matrix perturbation and optimal partition invariancy in linear optimization, Asia Pac. J. Oper. Res, Volume 32, Issue 03, (2015).

10. Goberna, M.A., Lopez, M.A.: Linear Semi-Infinite Optimization, Wiley, Chichester, England (1998).

11. Goberna, M.A., Lopez, M.A.: Post-Optimal Analysis in Linear Semi-Infinite Optimization, Springer Briefs, Springer, NY (2014).

12. Goberna, M.A., Lopez, M.A., Todorov, M.I.: On the stability of the feasible set in linear optimization, Set-Valued Analysis, 9, pp. 75-99 (2001).

13. Greenberg, H.J., Holder, A., Roos, C., Terlaky, T.: On the dimension of the set of rim perturbations for optimal partition invariance, SIAM J. Optim., 9, 207-216 (1998).

14. Hearn, D.W.: The gap function of a convex program, Oper. Res. Letters, 1, 67-71 (1982)

15. Jeyakumar, V., Wolkowicz, H.: Zero duality gaps in infinite-dimensional programming, J. Optim. Theory Appl., 67, pp. 87-108 (1990).

16. Lucchetti, R., Radrizzani, P., Villa, S.: Generic well posedness in Linear Programming, Pac. J. Optim., 4 (2008), pp. 513-525 (2008).

17. Martinez-Legaz, J.E., Volle, M.: Duality in D.C. Programming: The Case of Several D.C. Constraints, J. Math. Anal. Appl., 237 pp. 657-671 (1999)

18. Ochoa, P.D., Vera de Serio, V.N.: Stability of the primal-dual partition in linear semi-infinite programming, Optimization, 61, pp. 1449-1465 (2012).

19. Rockafellar, R.T.: Convex Analysis, Princeton U.P., Princeton (1970).

20. Rubinstein, G.S.: A comment on Voigt's paper "A duality theorem for linear semi-infinite programming", Optimization, 12 , pp. 31-32 (1981).

21. Todorov, M.: Necessary and sufficient conditions for global lower semicontinuity in linear semi-infinite optimization, Numer. Funct. Anal. Optim., 19, pp. 415-429 (1998).

22. Vinh, N.T., Kim, D.S., Tam, N.N., Yen, N.D.: Duality gap function in infinite dimensional linear programming, J. Math. Anal. Appl., 437, pp. 1-15 (2016). 